\title{
Tumor pardo espinal como primera manifestación de un hiperparatiroidismo primario: revisión sistemática de la literatura y reporte de un caso
}

\author{
Cristina Quevedo-Vélez ${ }^{1}$, Vanessa Castaño-Lopera ${ }^{1}$, Cristina Espinosa-De La Ossa ${ }^{2}$, \\ Jairo Giovanni Moncayo-Viveros ${ }^{3}$, Carlos Esteban Builes-Montaño ${ }^{4}$
}

${ }^{1}$ Médica y Cirujana, Hospital Pablo Tobón Uribe

${ }^{2}$ Médica y Cirujana, Corporación Universitaria del Caribe

${ }^{3}$ Médico especialista en medicina de Urgencias, Hospital Pablo

Tobón Uribe

${ }^{4}$ Médico Internista Endocrinólogo, Hospital Pablo Tobón Uribe Universidad de Antioquia

Autor responsable de la correspondencia con la revista ACE:

Carlos Esteban Builes-Montaño.

Dirección: Calle 78B \#69-240. Celular: 3053385136.cbuiles@

hptu.org.co

Notificación de conflictos: No existen conflictos de interés a declarar por parte de los autores.

Fecha de recepción: 28/03/2017

Fecha de aceptación: 23/06/2017

\section{Resumen}

$\mathrm{L}$ os tumores pardos son una forma rara de manifestación del hiperparatiroidismo; pueden afectar cualquier hueso del cuerpo como lesiones únicas o múltiples, pero es bastante inusual que afecten la columna. El compromiso espinal se acompaña de síntomas neurológicos severos, y en la mayoría de los casos reportados, se ha recurrido a la intervención neuroquirúrgica de la lesión para resolver este tipo de manifestaciones clínicas.

Presentamos el caso de un tumor pardo espinal, como primera manifestación de un hiperparatiroidismo primario, que fue tratado solo con paratiroidectomía y que no requirió ninguna intervención neuroquirúrgica para obtener una recuperación completa de los síntomas neurológicos.

La resección del tumor, busca mejorar los síntomas motores por compresión o establecer un diagnóstico histológico por sospecha de malignidad. Sin embargo, debido a que los tumores pardos en el hiperparatiroidismo primario ocurren por la acción de la paratohormona, estos podrían desaparecer completamente, normalizando los niveles de dicha hormona en sangre. En algunos casos se podría contemplar la posibilidad de no intervenir la lesión espinal y resolver inicialmente el hiperparatiroidismo.

\section{Abstract}

Brown tumors are a rare form of manifestation of a primary hyperparathyroidism; They can affect any bone in the body as single or multiple lesions, but the appearance of these lesions in the spine is quite unusual. When spinal involvement occurs, it is accompanied by severe neurological symptoms and in most of reported cases, the neurosurgical intervention of the lesion has been used to resolve this type of clinical manifestations.

We present the case of a spinal brown tumor as the first manifestation of a primary hyperparathyroidism that was treated with parathyroidectomy alone and did not require any neurosurgical intervention to obtain a complete recovery of the neurological symptoms.

Resection of the tumor, seeks to improve motor symptoms by compression or establish a histological diagnosis for suspected malignancy. However, because brown tumors occur due to the action of the paratohormone, a complete disappearance of the lesion could occur as the hormone levels normalizes. In some cases, the possibility of not intervening the spinal lesion could be contemplated and initially resolve the hyperparathyroidism.

Key Words: "Osteitis Fibrosa Cystica", "Primary Hyperparathyroidism" y "Bone Neoplasms"

\section{Introducción}

El hiperparatiroidismo primario (HPTP) es una de las endocrinopatías más frecuentes que afecta alrededor del 1\% de la población adulta, además, es la principal causa de hipercalcemia en el ámbito ambulatorio y hasta el $80 \%$ de los pacientes no presenta ningún síntoma al momento del diagnóstico ${ }^{(1,2)}$.

Las manifestaciones óseas del HPTP se reportan cada vez con menos frecuencia. En las primeras décadas del siglo XX, cuando se consideraba esta condición como una enfermedad poco frecuente, hasta el $70 \%$ de los pacientes tenían alguna alteración esquelética asociada ${ }^{(3)}$, como lo confirman los casos colombianos diagnosticados en la segunda mitad del siglo XX (3 A); sin embargo, con el cambio en la forma como se hace la medición de calcio y el uso de detectores multicanal a partir 
de la segunda mitad del siglo XX, el HPTP asintomático se ha hecho cada vez más prevalente y las manifestaciones esqueléticas se reportan en menos del $6 \%$ de los pacientes ${ }^{(1)}$.

Las manifestaciones esqueléticas más comunes del HPTP incluyen el dolor óseo, la baja densidad mineral y las fracturas por fragilidad. Es de anotar que estos pacientes usualmente se presentan con otras manifestaciones de la enfermedad como síntomas neuropsiquiátricos, manifestaciones renales y debilidad muscular. Los tumores pardos (TP) u osteoclastomas son una forma muy rara de manifestación del HPTP. Pueden afectar cualquier hueso del cuerpo como lesiones únicas o múltiples pero es bastante inusual que afecten la columna ${ }^{(4)}$. El compromiso espinal se acompaña de síntomas neurológicos severos y, la mayoría de las veces, se recurre a la intervención quirúrgica de la lesión espinal ${ }^{(5)}$.

Presentamos el caso de un tumor pardo espinal, como primera manifestación de un hiperparatiroidismo primario que fue tratado solo con paratiroidectomía.

\section{Presentación del caso}

Paciente masculino de 49 años, previamente sano, consulta inicialmente a un servicio de ortopedia por debilidad progresiva en los miembros inferiores, sin otros síntomas. Dentro de los estudios iniciales en una resonancia magnética de columna, se reporta una lesión a nivel de T2 y es llevado a una cirugía para descompresión de la columna con una sospecha de un tumor maligno en columna vs una metástasis de un primario por esclarecer. El estudio del tejido obtenido reporta "Fragmentos de tejido óseo constituido por espículas óseas maduras sin atipia, pero irregulares y comprometidos por un tumor compuesto por sábanas de células mononucleares epiteliodes de citoplasma eosinófilo claro bien definido, núcleos homogéneos de cromatina granular sin atipia ni mitosis, entremezclados con abundantes células gigantes multinucleados de tipo osteoclasto. Hay formación de osteoide. Lesión de células gigantes compatible con tumor óseo de células gigantes". Los síntomas empeoran después de la cirugía, con deterioro progresivo de la fuerza muscular. En el seguimiento ambulatorio tenía varias pruebas sanguíneas sin alteración, excepto por el valor de calcio sérico 13,5 mg/dl (referencia 8,5 a 10,4 $\mathrm{mg} / \mathrm{dl}$ ) y el reporte de una tomografía que mencionaba una lesión lítica en una de sus vértebras. Todo esto hace sospechar un mieloma múltiple. Un año después de la cirugía inicial, es remitido para una valoración por endocrinología en nuestra institución y, en ese momento, el examen neurológico revelaba una paraparesia sin pérdida del control de los esfínteres. Se decidió hospitalizarlo nuevamente y dentro de sus estudios iniciales (tabla 1) tenía una elevación de la PTH, el calcio sérico y la fosfatasa alcalina; en los rayos X simples se reportó una osteopenia generalizada sin lesiones óseas líticas, blásticas, ni quísticas y tampoco presentaba resorción de las falanges dis- tales ni de la clavícula; y en una ecografía de cuello se reportaba un posible adenoma de paratiroides inferior izquierdo. La nueva resonancia magnética de la columna reportaba una gran lesión a nivel de T2, que comprometía el cuerpo vertebral y los elementos posteriores, principalmente en el lado izquierdo, con disminución severa en la amplitud del canal central y compresión del saco tecal, del cordón medular, mielopatía compresiva y estenosis foraminal bilateral T2-T3 de predominio izquierdo. La lesión es descrita de característica Isointensa en secuencia T1 e hiperintensa en T2. Se le propuso al paciente una nueva cirugía de descompresión de la columna, fijación y resección de la masa, a la cual se negó por temor a los riesgos. Se decidió entonces realizar la cirugía de resección del adenoma paratiroideo. Después de ésta se normalizaron los niveles de calcio y se disminuyó de manera sustancial la PTH; y al tercer día posoperatorio, el paciente presentaba una marcada mejoría de la fuerza muscular con recuperación completa de la marcha.

Tabla 1. Exámenes de laboratorio iniciales del paciente

\begin{tabular}{l|c}
\hline \multicolumn{1}{c|}{ PRUEBA } & VALOR \\
\hline Calcio & $13,6 \mathrm{mg} / \mathrm{dl}(\mathrm{VR} 8,5-10,4)$ \\
\hline Fósforo & $2,7 \mathrm{mg} / \mathrm{dl}(\mathrm{VR}(2,3-4-6)$ \\
\hline Paratohormona & $651 \mathrm{pg} / \mathrm{ml}(\mathrm{VR}(10-55)$ \\
\hline Fosfatasa alcalina & $225 \mathrm{UI} / \mathrm{mL}(\mathrm{VR}(68-240)$ \\
\hline
\end{tabular}

\section{Revisión sistemática de la literatura}

Se realizó una revisión de la literatura en Pubmed, EMBASE, Scielo y Google Scholar con las palabras claves "Osteitis Fibrosa Cystica", "Primary Hyperparathyroidism" y "Bone Neoplasms" encontrando 24 casos reportados en inglés y francés de tumores pardos como manifestación inicial de un hiperparatiroidismo primario ${ }^{(5-25)}$ (tabla 2).

La mayoría de reportes se han hecho en mujeres entre la cuarta y séptima década de la vida. Se pudieron obtener datos del perfil metabólico del calcio parcial en el $80 \%$ de los casos y total en el 30\%. La mayoría de los pacientes en quienes se obtuvo el valor de calcio presentaban hipercalcemia al momento del diagnóstico un $60 \%$ de ellos con valores superiores a $13 \mathrm{mg} / \mathrm{dl}$, un $30 \%$ con valores superiores a $14 \mathrm{mg} / \mathrm{dl}$; la hipercalcemia se acompañaba, en una proporción importante, de pacientes de hipofosfatemia (85\%) y el 36\% de los pacientes se presentaron con niveles de paratohormona superior a $1.000 \mathrm{pg} / \mathrm{ml}$.

Casi todos los pacientes presentaron algún síntoma asociado al tumor (92\%) siendo el más frecuente el compromiso motor por compresión. La gran mayoría se recuperaron completamente después de la intervención. La resección del tumor pardo acompañada de paratiroidectomía fue la forma de tratamiento más común, algunos pacientes fueron tratados solo con paratiroidectomía y, de éstos, solo uno tenía síntomas 
Tabla 2. Revisión sistemática de la literatura de tumores pardos espinales

\begin{tabular}{|c|c|c|c|}
\hline \multicolumn{4}{|c|}{ Características generales } \\
\hline Edad en años, mediana (rango) & \multicolumn{3}{|c|}{$49(16-69)$} \\
\hline Mujeres & \multicolumn{3}{|c|}{$16(66 \%)$} \\
\hline Calcio, media (DS) & \multicolumn{3}{|c|}{$13,1 \pm 1,4 \mathrm{mg} / \mathrm{dl}$} \\
\hline Fósforo, media (DS) & \multicolumn{3}{|c|}{$2,4 \pm 0,8 \mathrm{mg} / \mathrm{dl}$} \\
\hline Paratohormona, mediana (rango) & \multicolumn{3}{|c|}{$948(50-3200) \mathrm{pg} / \mathrm{ml}$} \\
\hline Fosfatasa alcalina, mediana (rango) & \multicolumn{3}{|c|}{$1.198(127-6.661) \mathrm{UI} / \mathrm{ml}$} \\
\hline \multicolumn{4}{|c|}{ Síntomas al momento de presentación } \\
\hline Síntomas motores & \multicolumn{3}{|c|}{$15(60 \%)$} \\
\hline Compromiso de esfínteres & \multicolumn{3}{|c|}{$6(24 \%)$} \\
\hline Radiculopatia & \multicolumn{3}{|c|}{$10(40 \%)$} \\
\hline Dolor local en el sitio del tumor & \multicolumn{3}{|c|}{$9(36 \%)$} \\
\hline \multicolumn{4}{|c|}{ Nivel espinal afectado } \\
\hline Cervical & \multicolumn{3}{|c|}{$3(12,5 \%)$} \\
\hline Dorsal & \multicolumn{3}{|c|}{$2(8 \%)$} \\
\hline Lumbar & \multicolumn{3}{|c|}{$12(50 \%)$} \\
\hline Sacro & \multicolumn{3}{|c|}{$7(29 \%)$} \\
\hline \multicolumn{4}{|c|}{ Tratamiento } \\
\hline Resección del tumor+ paratiroidectomía & \multicolumn{3}{|c|}{$16(66 \%)$} \\
\hline Solo paratiroidectomía & \multicolumn{3}{|c|}{$6(25 \%)$} \\
\hline Solo resección de la masa & \multicolumn{3}{|c|}{$2(8 \%)$} \\
\hline \multicolumn{4}{|c|}{ Resultado del tratamiento } \\
\hline Recuperación completa & \multicolumn{3}{|c|}{$22(92 \%)$} \\
\hline \multirow[t]{2}{*}{ Persistencia de los síntomas } & \multicolumn{3}{|c|}{$2(8 \%)$} \\
\hline & $\begin{array}{c}\text { Resección del tumor + } \\
\text { paratiroidectomía }\end{array}$ & Paratiroidectomía & Resección del tumor \\
\hline Recuperación completa & $14(88 \%)$ & $6(100 \%)$ & $2(100 \%)$ \\
\hline Persistencia de los síntomas & $2(12 \%)$ & 0 & 0 \\
\hline
\end{tabular}

compresivos. La gran mayoría de los pacientes presentaron recuperación completa de los síntomas después de la intervención, independientemente del tipo.

\section{Discusión}

Los niveles aumentados de PTH, independientemente de su causa, producen un aumento en la actividad osteoclástica, promoviendo la resorción y la fibrosis ósea, y esto a su vez, ocasiona una disminución generalizada del contenido mineral y la aparición de lesiones quísticas, condición que en su máxima expresión se denomina Osteítis Fibrosa Quística (OFQ). Los tumores pardos (TP) son una forma localizada de la OFQ que ocurren por la estimulación constante de la PTH sobre los osteoblastos, promoviendo la activación y la diferenciación de los osteoclastos, y su confluencia en células gigantes multinu- cleadas, que generan destrucción local con áreas hemorrágicas y depósitos de hemosiderina en su interior, de donde obtienen su nombre ${ }^{(26)}$.

Los TP espinales son una forma de manifestación inicial bastante inusual del HPTP que afecta aproximadamente a un 3,0\%-4,5\% de los pacientes con HPTP y un 1,5\%-1,7\% de los HPT secundarios (HPTS) ${ }^{(27)}$. Los TP espinales parecen seguir la misma distribución epidemiológica, presentándose con mayor frecuencia en las mujeres posmenopáusicas ${ }^{(1)}$ y afectando la columna dorsal de manera predominante.

La hipercalcemia es un hallazgo frecuente entre los pacientes con HPTP y TP espinales y hasta el 30\% de los reportes cumplirían con el criterio propuesto de hiperparatiroidismo primario agudo con valores de calcio superior a $14 \mathrm{mg} / \mathrm{dl}^{(28)}$. Adicionalmente, una proporción importante de estos pacientes cursa con valores bastante elevados de PTH lo cual podría 
hacer pensar en un carcinoma paratiroideo ${ }^{(29)}$; sin embargo, en ninguno de los casos ésta fue la causa del HPTP.

Los tumores pardos pueden cursar con un amplio espectro de manifestaciones clínicas, desde un estado asintomático hasta cursar con un cuadro severo como el síndrome de compresión medular, como consecuencia del crecimiento del tumor. Los síntomas varían según su localización. En aquellos que comprometen las vértebras predomina el dolor axial y podrían manifestarse con mielopatía, radiculopatía, síndrome de cauda equina, alteración en el control de esfínteres y aparición de fracturas patológicas.

Dentro de los casos reportados, es usual que se realizaran múltiples estudios para enfocar la lesión entre los cuales se encuentran: radiografías en las que se observan lesiones líticas únicas o múltiples mal demarcadas en expansión, además de desmineralización generalizada. Por otro lado, la tomografía muestra mejor definición de la erosión local y extensión presentada no solo en hueso sino también en tejido blando, que se intensifica con el medio de contraste; sin embargo, no es específica para diagnosticar este tipo de tumores, porque no los distingue de otro tipo de lesiones. Por último, la Resonancia Magnética es de gran ayuda en la mejor caracterización de las lesiones y su extensión, éstas se ven usualmente hipointensas en la secuencia de T1, hiper o hipointensas en la secuencia de T2 (esto se lo debe al grado de su componente quístico), con realce posterior a la administración del medio de contraste por su alta vascularización, la formación de hemorragias intratumorales que generan niveles líquido-líquido. El compromiso en columna se caracteriza por ser de componente sólido, que frecuentemente causa destrucción de la corteza y extensión a tejidos blandos (figuras 1 al 4) ${ }^{(5)}$.

La biopsia se ha considerado como la prueba de oro para el diagnóstico, y las lesiones se describen como un tumor no encapsulado que se caracteriza por un estroma fibrovascular sobrepuesto a numerosas células gigantes multinucleadas. También se describen macrófagos con hemosiderina en su interior, material de calcificación y fibroblastos en ocasiones. Histológicamente, no es diferente en su morfología a otras lesiones constituidas por células gigantes, lo que genera una dificultad al momento de realizar diagnósticos diferenciales ${ }^{(23)}$.

En la gran mayoría de casos de TP espinales, el tratamiento ha sido la resección del tumor, bien sea buscando mejorar los síntomas motores por compresión, para establecer un diagnóstico histológico o por sospecha de algún tipo de malignidad. Sin embargo, debido a que los tumores son una neoplasia que ocurre por la proliferación y la fusión de osteoclastos debido a la acción de la paratohormona, éstos regresan completamente después de su normalización. Es por esto que en algunos casos podría considerarse no intervenir la lesión espinal y esperar una recuperación completa del paciente ${ }^{(10)}$, como en el caso reportado.

Figura 1. Corte transversal con secuencia T2: se observa lesión que compromete el cuerpo vertebral y los elementos posteriores principalmente del lado izquierdo, causando disminución severa del canal central con compromiso del saco tecal, cordón medular y mielopatía compresiva

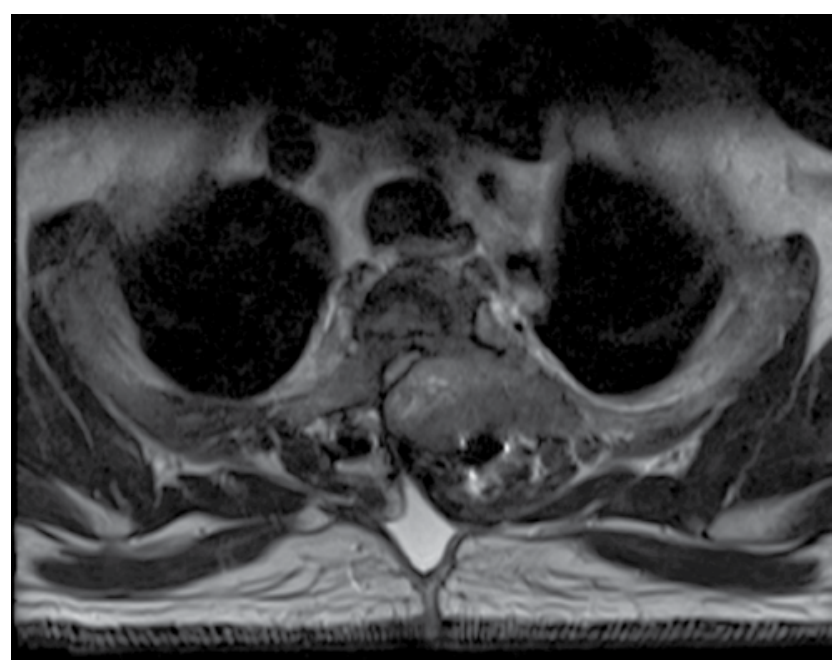

Figura 2. Secuencia T1: imagen de la derecha sin contraste, imagen de la izquierda con contraste. Nótese cómo la masa se realza con medio de contraste, lo que evidencia interrupción de la membrana basal

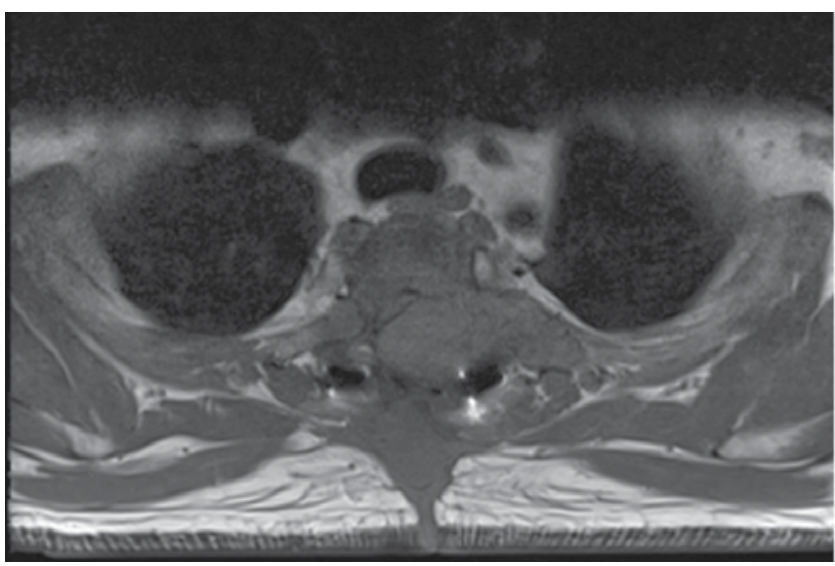

http://revistaendocrino.org/

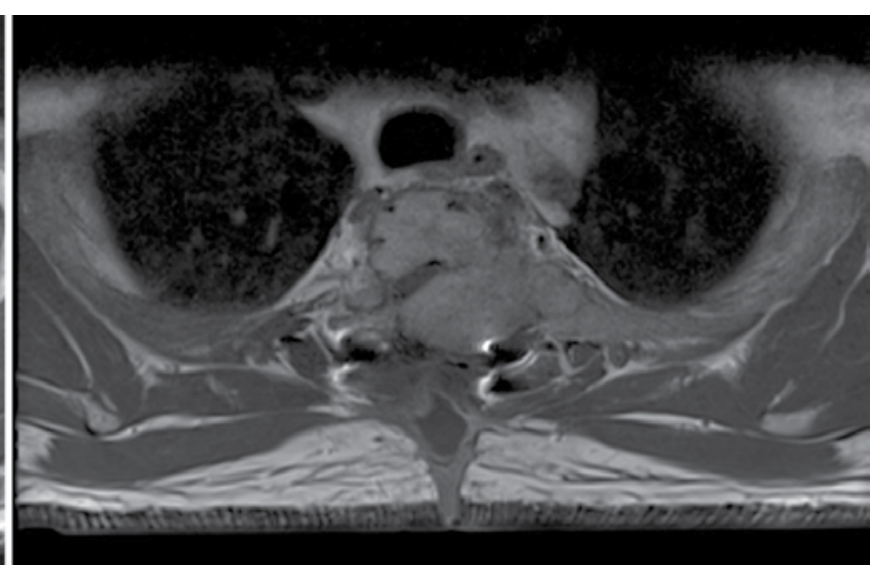


Figura 3. Imagen de la izquierda: secuencia T1. Imagen de la derecha: secuencia T2. Ambas sin contraste, corte sagital. En las imágenes se aprecia lesión que causa disminución de la amplitud del canal vertebral y compresión del canal medular, que se ve con mayor exactitud en secuencia $\mathrm{T} 2$, donde se resalta el espacio medular. Además, se observa, cambios en la altura del cuerpo vertebral
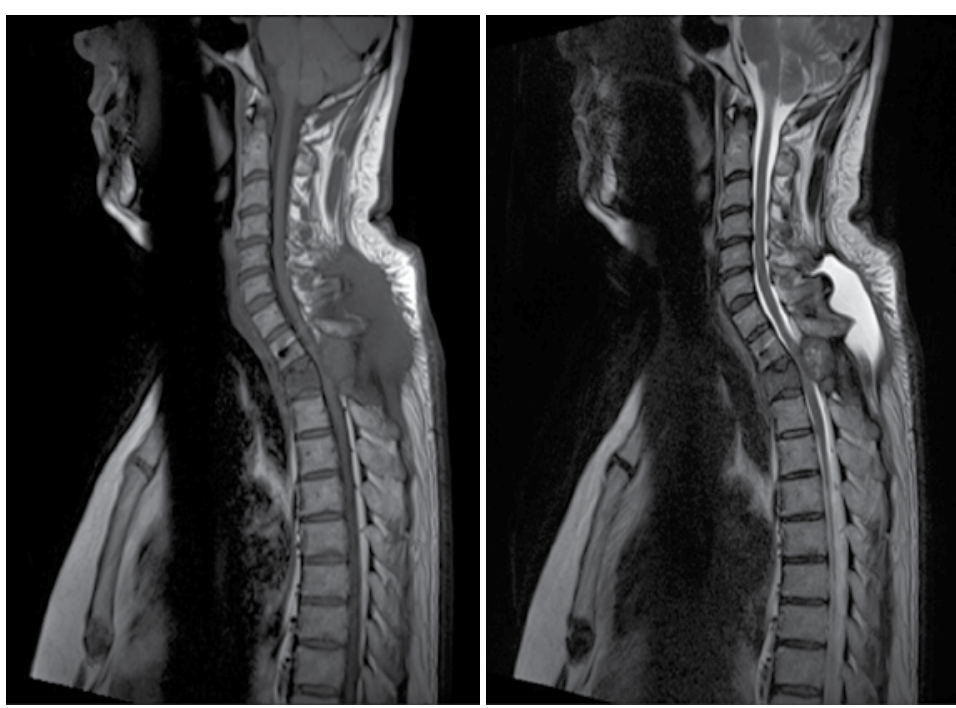

Figura 4. Secuencia STIR: supresión de la grasa; se aprecia que la masa no desaparece en esta proyección, lo que demuestra que la masa no es de componente graso

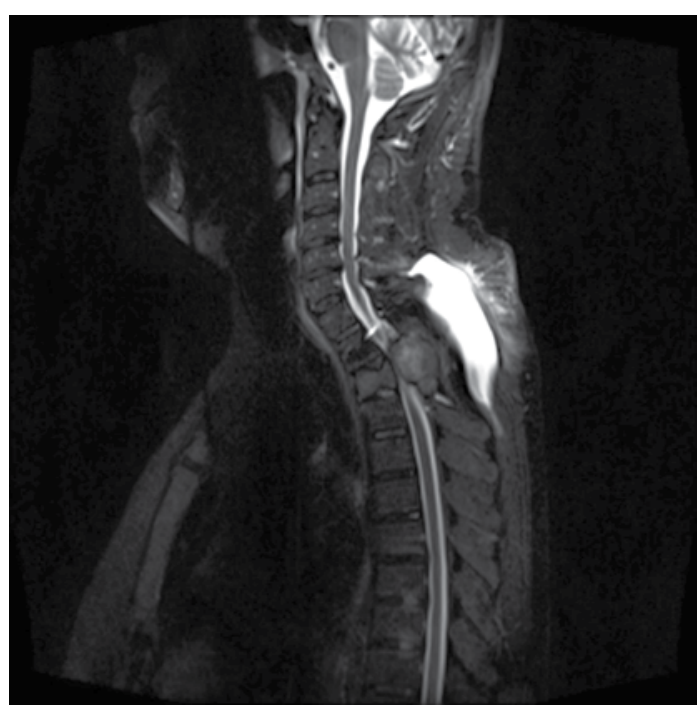

\section{Referencias}

1. Eufrazino C, Veras A, Bandeira F. Epidemiology of Primary Hyperparathyroidism and its Non-classical Manifestations in the City of Recife, Brazil. Clin Med Insights Endocrinol Diabetes. 2013;6:69-74.

2. Gasser RW. Clinical aspects of primary hyperparathyroidism: clinical manifestations, diagnosis, and therapy. Wien Med Wochenschr. 2013;163(1718):397-402.

3. Albright F, Aub JC, Bauer W. Hyperparathyroidism: A common and polymorphic condition as illustrated by seventeen proved cases from one clinic. Journal of the American Medical Association. 1934;102(16):1276-87.

3.A. Jácome-Roca A. Historia de las glándulas partatiroides. Rev Col Endocrinol Diab Metab 2016; 3 (3): 11-24.

4. Pappu R, Jabbour SA, Regianto AM, Reginato AJ. Musculoskeletal manifestations of primary hyperparathyroidism. Clin Rheumatol. 2016;35(12):3081-7.

5. Sonmez E, Tezcaner T, Coven I, Terzi A. Brown Tumor of the Thoracic Spine: First Manifestation of Primary Hyperparathyroidism. J Korean Neurosurg Soc. 2015;58(4):389-92.

6. Shaw JE, Sicree RA, Zimmet PZ. Global estimates of the prevalence of diabetes for 2010 and 2030. Diabetes Res Clin Pract. 2010;87(1):4-14

7. Shuangshoti S, Hongsaprabhas C, Chandraprasert S, Rajatapiti B. Parathyroid adenoma, brown tumor and cauda equina compression. J Med Assoc Thai. 1972;55(4):251-8.

8. Sundaram M, Scholz C. Primary hyperparathyroidism presenting with acute paraplegia. AJR Am J Roentgenol. 1977;128(4):674-6.

9. Siu K, Sundaram M, Schultz C, Kirwan L. Primary hyperparathyroidism presenting as spinal cord compression: report of a case. Australian and New Zealand Journal of Surgery. 1977;47(5):668-72.

10. Ganesh A, Kurian S, John L. Complete recovery of spinal cord compression following parathyroidectomy. Postgrad Med J. 1981;57(672):652-3.

11. Yokota N, Kuribayashi T, Nagamine M, Tanaka M, Matsukura S, Wakisaka S. Paraplegia caused by brown tumor in primary hyperparathyroidism. Case report. J Neurosurg. 1989;71(3):446-8.

12. Daras M, Georgakopoulos T, Avdelidis D, Gravani A, Tuchman AJ. Spinal cord compression in primary hyperparathyroidism. Report of a case and review of the literature. Spine (Phila Pa 1976). 1990;15(3):238-40.

13. Kashkari S, Kelly TR, Bethem D, Pepe RG. Osteitis fibrosa cystica (brown tumor) of the spine with cord compression: Report of a case with needle aspiration biopsy findings. Diagnostic Cytopathology. 1990;6(5):349-53.

14. Sarda AK, Arunabh, Vijayaraghavan M, Kapur M. Paraplegia due to osteitis fibrosa secondary to primary hyperparathyroidism: Report of a case. Surgery Today. 1993;23(11):1003-5.
15. Motateanu M, Déruaz JP, Fankhauser H. Spinal tumour due to primary hyperparathyroidism causing sciatica: case report. Neuroradiology. 1994;36(2):134-6.

16. Ashebu SD, Dahniya MH, Muhtaseb SA, Aduh P. Unusual florid skeletal manifestations of primary hyperparathyroidism. Skeletal Radiol. 2002;31(12):720-3.

17. Mustonen AO, Kiuru MJ, Stahls A, Bohling T, Kivioja A, Koskinen SK. Radicular lower extremity pain as the first symptom of primary hyperparathyroidism. Skeletal Radiol. 2004;33(8):467-72.

18. Altan L, Kurtoglu Z, Yalcinkaya U, Aydinli U, Erturk E. Brown tumor of the sacral spine in a patient with low-back pain. Rheumatol Int. 2007;28(1):77-81.

19. Haddad FH, Malkawi OM, Sharbaji AA, Jbara IF, Rihani HR. Primary hyperparathyroidism. A rare cause of spinal cord compression. Saudi Med J. 2007;28(5):783-6.

20. Khalil PN, Heining SM, Huss R, Ihrler S, Siebeck M, Hallfeldt K, et al. Natura history and surgical treatment of brown tumor lesions at various sites in refractory primary hyperparathyroidism. Eur J Med Res. 2007;12(5):222-30.

21. Hoshi M, Takami M, Kajikawa M, Teramura K, Okamoto T, Yanagida I, et al. A case of multiple skeletal lesions of brown tumors, mimicking carcinoma metastases. Arch Orthop Trauma Surg. 2008;128(2):149-54.

22. Lee JH, Chung SM, Kim HS. Osteitis fibrosa cystica mistaken for malignant dis ease. Clin Exp Otorhinolaryngol. 2013;6(2):110-3.

23. Khalatbari MR, Moharamzad Y. Brown tumor of the spine in patients with primary hyperparathyroidism. Spine (Phila Pa 1976). 2014;39(18):E1073-9.

24. Alfawareh MD, Halawani MM, Attia WI, Almusrea KN. Brown tumor of the cervical spines: a case report with literature review. Asian Spine J. 2015;9(1):110-20

25. Andia A, Inès M, Mahamane Sani MA, Brah S, Sani Beydou S, Daou M, et al. Lomboradiculagie révélatrice d'une tumeur brune secondaire à une hyperparathyroïdie primaire : à propos d'un cas et revue de la littérature2015.

26. Chavin HC, Pisarevsky AA, Chavin C, Koll L, Petrucci EA. [Brown tumor as the initial manifestation of primary hyperparathyroidism]. Medicina (B Aires). 2008;68(3):219-21.

27. Arsalanizadeh B, Westacott R. Osteoclastomas ('brown tumours') and spinal cord compression: a review. Clin Kidney J. 2013;6(2):220-3.

28. Cundy T, Darby AJ, Berry HE, Parsons V. Bone metabolism in acute parathyroid crisis. Clin Endocrinol (Oxf). 1985;22(6):787-93.

29. Wei $\mathrm{CH}$, Harari A. Parathyroid carcinoma: update and guidelines for management. Curr Treat Options Oncol. 2012;13(1):11-23. 